\title{
Erratum to: prevalence of anelloviruses (TTV, TTMDV, and TTMV) in healthy blood donors and in patients infected with HBV or HCV in Qatar
}

Ahmed A. Al-Qahtani ${ }^{1,2,3}$, Enas S. Alabsi ${ }^{4,5}$, Raed AbuOdeh ${ }^{6}$, Lukman Thalib ${ }^{4}$, Mohamed E. El Zowalaty ${ }^{7}$ and Gheyath K. Nasrallah ${ }^{4,5^{*}}$

\section{Erratum}

Following publication of this article [1] it has come to our attention that Dr. Mohamed El Zowalaty should have been listed as a contributing author having been involved in the data entry, figure construction, writing and revision of manuscript and table generation. The original version has been revised to reflect this.

\begin{abstract}
Author details
${ }^{1}$ Department of Infection and Immunity, Research Center, King Faisal Specialist Hospital and Research Center, Riyadh, Saudi Arabia. ${ }^{2}$ Department of Microbiology and Immunology, Alfaisal University School of Medicine, Riyadh, Saudi Arabia. ${ }^{3}$ Liver Disease Research Center, King Saud University, Riyadh, Saudi Arabia. ${ }^{4}$ Department Health Sciences, College of Arts and Sciences, Qatar University, PO Box 2713, Doha, Qatar. ${ }^{5}$ Biomedical Research Center, Qatar University, Doha, Qatar. ${ }^{6}$ Department of Medical Laboratory Sciences, University of Sharjah, Sharjah, UAE. 'Virology and Microbiology Research Laboratory \& Antibiotic Research Unit, School of Health Sciences, University of KwaZulu Natal, University Rd, Westville Campus, Durban 4000, South Africa.
\end{abstract}

Received: 22 June 2017 Accepted: 23 June 2017

Published online: 27 July 2017

\section{Reference}

1. Al-Qahtani A, Alabsi E, AbuOdeh R, Thalib L, Nasrallah G. Prevalence of anelloviruses (TTV, TTMDV, and TTMV) in healthy blood donors and in patients infected with HBV or HCV in Qatar. Virology Journal. 2016; 13(1). DOI:10.1186/s12985-016-0664-6.

\footnotetext{
* Correspondence: gheyath.nasrallah@qu.edu.qa

${ }^{4}$ Department Health Sciences, College of Arts and Sciences, Qatar University, PO Box 2713, Doha, Qatar

${ }^{5}$ Biomedical Research Center, Qatar University, Doha, Qatar
} 